Running head: Pupil dilation reveals attentional load

Manuscript accepted for publication in "Biological Psychology"

\title{
Pupil dilation reveals top-down attentional load during spatial monitoring
}

\author{
Matteo Lisi ${ }^{1}$ \\ Mario Bonato $^{2}$ \\ Marco Zorzi ${ }^{3,4}$
}

1 Laboratoire Psychologie de la Perception, Université Paris Descartes \& CNRS UMR 8242,

Paris, France

2 Department of Experimental Psychology, Ghent University, Ghent, Belgium

3 Department of General Psychology and Center for Cognitive Neuroscience, University of Padova, Padua, Italy

4 IRCCS San Camillo Hospital, Lido Venice, Italy

Correspondence concerning this article should be addressed to Matteo Lisi, Laboratoire Psychologie de la Perception, Université Paris Descartes, 45 rue Des Sant-Pères, 75006, Paris, France. E-mail: matteo.lisi@parisdescartes.fr 


\begin{abstract}
It has long been known that the diameter of human pupil enlarges with increasing effort during the execution of a task. This has been observed not only for purely mechanical effort but also for mental effort, as for example the computation of arithmetic problems with different levels of difficulty. Here we show that pupil dilation reflects changes in visuospatial awareness induced by attentional load during multi-tasking. In the single-task condition, participants had to report the position of lateralized, briefly presented, masked visual targets ("right", "left", or "both" sides). In the multitasking conditions, participants also performed additional tasks, either visual or auditory, to increase the attentional load. Sensory stimulation was kept constant across all conditions to rule out the influence of low-level factors. Results show that event-related pupil dilation strikingly increased with task demands, mirroring a concurrent decrease in visuospatial awareness. Importantly, pupil dilation significantly differed between two dual-task conditions that required to process the same number of stimuli but yielded differed levels of accuracy (difficulty). In contrast, pupil dilation did not differ between two conditions which were equally challenging but differed both in the modality of the dual task (auditory vs. visual) and in the number of stimuli to be attended. We conclude that pupil dilation genuinely reflects the top-down allocation of supramodal attentional resources.
\end{abstract}




\section{Introduction}

The measurement of pupil diameter (i.e., 'pupillometry') has been used in psychology for more than 50 years as a psychophysiological index of processing load and resource allocation (Hess \& Polt, 1960; Hess \& Polt, 1964; Kahneman \& Beatty, 1966). Increased pupil diameter (dilation/mydriasis) is associated with greater effort, and this has been observed both for purely mechanical effort (e.g. picking up different weights, see Nunnally et al., 1967) and for mental effort (e.g. processing arithmetic problems with different levels of difficulty, see Ahern \& Beatty, 1979). The degree of pupil dilation has been shown to reflect also the difficulty of lower-level, more sensorial, tasks, such as the discrimination between tones of different pitch (Kahneman \& Beatty, 1967). Accordingly, given the sensitivity of pupil diameter to processing load, Kahneman (1973) used it as the primary measure of processing load in his effort theory of attention, suggesting that it could provide a window on the "intensive" aspects of attention, intended as distinct from the more often studied "selective" aspects. More recently, changes in pupil size have been suggested to reflect not only attentional allocation but also a broad range of processes involved in cognitive control (Laeng, Ørbo, Holmlund, \& Miozzo, 2011; Brown, Kindermann, Siegle, Granholm, Wong, \& Buxton, 1999; van Steenbergen \& Band, 2013).

Which mechanism links the size of the pupil to mental effort? It is worth remembering that the size of the pupil is determined primarily by the exposure to light and the accommodation reflex. Changes in illumination can elicit pupil dilation up to a maximum of more than double of its typical size (about 3mm, MacLachlan \& Howland, 2002). Changes in pupil size reflecting cognitive processes are instead much smaller (often less that $0.5 \mathrm{~mm}$ ). These changes can be extracted by performing time-locked averaging with respect to the event of interest, and are often normalized with respect to pupil size at baseline, tipically measured before the onset of the event (Beatty \& Lucero-Wagoner, 2000; Beatty, 1982). These cognitively-related pupil dilation responses have been recently linked to a neurotransmitter system, the locus coeruleus-noradrenergic (LC-NE) neuromodulatory system (Aston-Jones \& Cohen, 2005). Specifically, it has been shown that pupil size correlates with tonic activity of locus coeruleus (LC) in several non-human species (AstonJones \& Cohen, 2005), and that pupil size in humans correlates with behaviors that have been linked with LC-NE activity in a variety of tasks (Gilzenrat, Nieuwenhuis, Jepma, \& Cohen, 2010; Jepma \& Nieuwenhuis, 2011; Murphy, Robertson, Balsters, \& O'Connell, 2011). Because the LC is the sole source of the neurotransmitter norepinephrine (NE) in the brain, it has been suggested that activity within the NE system is reflected in the dilation of the eye's pupil, which in turn would offer a unique window on the NE system activity (Laeng, Sirois, \& Gredeback, 2012).

The LC-NE system has widespread connections and is thought to be involved in many cognitive functions, including attention and alertness (Petersen \& Posner, 2012; Posner \& Petersen, 1990). One recent hypothesis ascribes to the LC-NE system a central role in the functional integration of attentional networks (Corbetta, Patel, \& Shulman, 2008). Another theory, the adaptive gain theory (Aston-Jones \& Cohen, 2005), maintains that the LC-NE system modulates neural gain (responsivity) thorough the brain. It is thought to have two modes of activity, phasic and tonic (or more likely a continuum between these two modes), corresponding to high or low neural gain. In the phasic mode, LC cells exhibit large phasic activations, typically related to the processing of task-relevant stimuli. This mode of functioning is usually associated with higher levels of task engagement and performance (Aston-Jones, Rajkowski, \& Cohen, 1999; Aston-Jones, Rajkowski, Kubiak, \& Alexinsky, 1994; Gabay, Pertzov, \& Henik, 2011; Murphy, Robertson, Balsters, \& O'Connell, 2011; Usher, 1999). Conversely, in the tonic mode, LC cells show smaller, sporadic phasic activations, but higher tonic activity. This is associated with increased distractibility and poorer performance in tasks that require focused attention. Nevertheless, the tonic mode leads to increased sensitivity in detecting novel or unexpected stimuli, which can be advantageous in some environmental contexts. The theory thus proposes a crucial role for the LC-NE system in regulating task engagement and behavioral flexibility according to different environmental contingencies (Aston-Jones \& Cohen, 2005). Importantly, the adaptive gain theory predicts larger phasic 
responses in LC activity when subjects engage in a demanding task, which are accompanied by larger pupil dilations (Gabay et al., 2011).

In the present study we investigated whether the amplitude of pupil dilation could reflect the level of 'pure' top-down attentional load, as opposed to experimental manipulations where attentional load increases as a function of the number of stimuli presented, or of the physical similarity of the stimuli to be discriminated (e.g., Ahern \& Beatty, 1979; Beatty, 1982; Kahneman $\&$ Beatty, 1966, 1967). For this purpose, we designed an experimental paradigm characterized by a dual-task manipulation during a spatial monitoring paradigm. Our approach is based on a method that was proven to induce striking contralesional deficits of visual awareness in patients with unilateral brain damage (Bonato, Priftis, Marenzi, Umiltà, \& Zorzi, 2010; Bonato, 2015). Two features make this approach particularly suitable for measuring event-related pupillary responses. The first is that stimuli are kept identical across task conditions with different levels of attentional load (Bonato, 2012; also see Bonato, Spironelli, Lisi, Priftis, \& Zorzi, 2015, where this feature is exploited in a ERP study). The second feature is that patients' spatial monitoring performance is characterized by similar rates of contralesional omissions across very different dual tasks (Bonato, Priftis, Umiltà, \& Zorzi, 2013), suggesting the engagement of unspecific, supramodal attentional resources.

Attentional load has been shown to interfere with visuospatial orienting also in healthy subjects, although to a much lesser extent (Bonato et al., 2015; Dodds et al., 2008; O'Connell, Schneider, Hester, Mattingley, \& Bellgrove, 2011; Peers, Cusack, \& Duncan, 2006; Pérez et al., 2009). Following Bonato et al. (2010), in the present study we assessed the effect of attentional load on visuospatial awareness by means of a primary spatial task, common to all the conditions, which consisted in verbally reporting the position of lateralized, briefly-presented, masked targets that could appear either on the left, on the right, or on both sides. The aims of our study were twofold: first, we sought to determine whether pupil dilation provides an index of 'pure' top-down attentional load in healthy participants and, second, whether pupil dilation correlates with behavioural performance in the visuospatial monitoring task.

\section{Methods}

Participants. Twenty-four participants (mean age 23.3 years, range 19-29 years, 15 females) participated in the study. All participants signed a written informed consent and had normal or corrected to normal visual acuity. The study was approved by the local Ethics Committee (Department of General Psychology, University of Padova).

Apparatus. The experiment was conducted in a quiet and dimly lit room. The only sources of light were the computer screen and a lamp (tungsten incandescent light bulb, 25W/375lm) placed immediately behind the monitor and directed toward the wall. Participants were seated with the head positioned on a chin rest at a distance of $60 \mathrm{~cm}$ from the computer screen. The experiment was run on a PC, using E-Prime 2.0 software (Psychology Software Tools, Pittsburgh, PA). Eye movements were recorded with a sampling frequency of $60 \mathrm{~Hz}$ through a Tobii T120 screen-based eyetracker (Tobii Technology, Sweeden), which was used also to present stimuli through its embedded 17-inch TFT monitor.

Stimuli. Visual stimuli were presented on a gray background $(31.5 \mathrm{~cd} / \mathrm{m} 2)$ and consisted of lateralized targets for the primary spatial task, plus a colored shape appearing at fixation and a brief binaural sound presented through headphones for the secondary task(s). The lateralized targets (Fig. 1 , panel A) consisted in black $(0.33 \mathrm{~cd} / \mathrm{m} 2)$ dots of $0.75^{\circ}$ of diameter, appearing either on the left, on the right, or both sides of the screen (eccentricity $14^{\circ}$ ). Their duration was calibrated according to individual sensitivity (see Procedure below). The shape presented at fixation was either a square, a rhombus or a circle (matched area), filled in by one out of three colors (orange, green, blue), resulting in 9 different color-shape combinations (see Fig. 1, panel B). The sound was a $100 \mathrm{~ms}$ pure tone (three frequencies equally spaced in log-units were used: high, $796 \mathrm{~Hz}$, medium, $450 \mathrm{~Hz}$ and low pitch, $250 \mathrm{~Hz}$ ). After the disappearance of the targets, two masks (one for each side, left 
and right) were presented ( 4 black dots, arranged as the corners of a square centered on target position; side $1.8^{\circ}$ ).

Procedure. The experiment consisted in 4 experimental blocks (81 trials each) plus a pre-test block, to be completed in one session, lasting approximately one hour. Each trial (Fig. 1, panel A) started with the presentation of a black fixation cross in the center of the screen. Gaze contingency was then implemented: targets were concurrently presented $800 \mathrm{~ms}$ after participants maintained gaze within an area of $2^{\circ}$ around the center of the screen for at least $200 \mathrm{~ms}$ consecutively. Shortly after the presentation of the targets, the lateralized mask appeared on both sides, simultaneously with the fixation cross which replaced the central shape. One second later, a question mark appeared in the middle of the screen to prompt the participant to vocally report the target stimuli according to task instructions.

While the stimuli were always identical, the instructions changed across the four experimental blocks, thereby yielding four different task conditions. Each participant performed: i) a single task (single), where only the position of the lateralized targets had to be reported (left, right, both sides); ii) a visual dual-task (color), which required to report target position and the color of the central shape (green, blue or orange); iii) an auditory dual-task (sound), which required to report target position and the pitch of the binaural sound (high, medium or low pitch); and iv) a visual triple-task (shape-color), which required the participant to report target position and both the color and the type of central shape (square, rhombus or circle). The experimenter coded participants' responses online through a computer keyboard. The order of the four blocks (i.e., task conditions) was counterbalanced across participants.

The duration of visual stimuli was adapted to individual sensitivity in a pre-test block (single task condition), through a brief (54 trials) weighted up-down staircase procedure (Kaernbach, 1991) with a target performance $\sim 75 \%$ correct responses. This allowed to roughly equate the difficulty of the primary task across participants. The average duration was $161 \mathrm{~ms}$ (standard deviation $88 \mathrm{~ms}$, range $67-317 \mathrm{~ms})$.

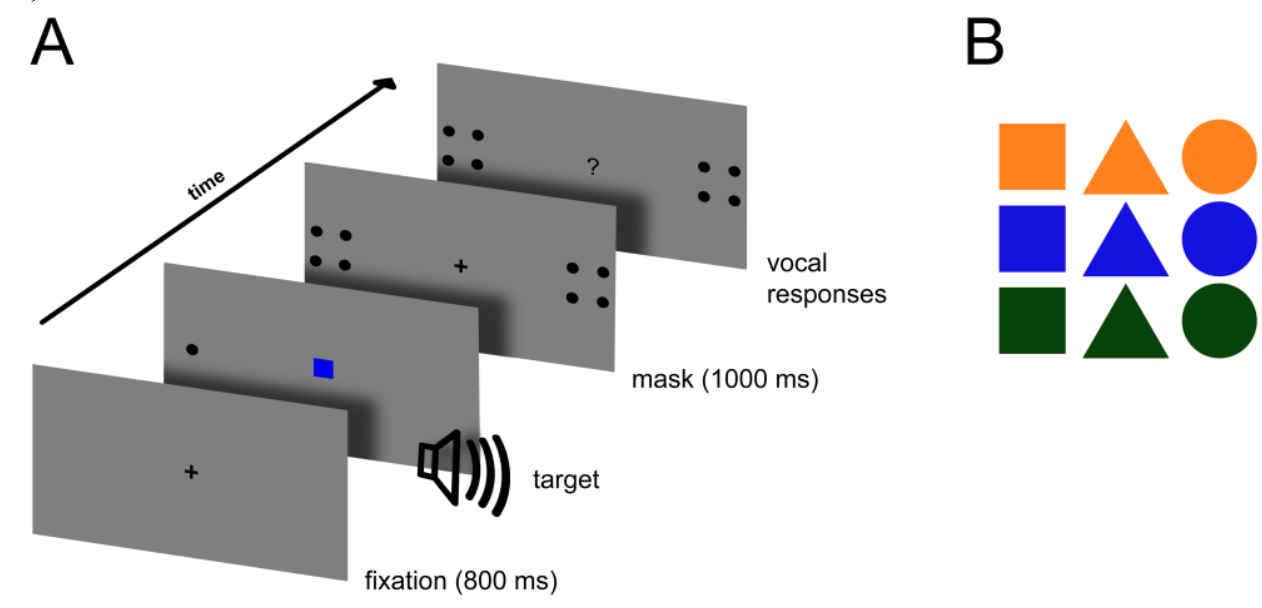

Fig. 1 (A) Schematic representation of an experimental trial (left target). (B) The possible combinations of color and shape for the central stimulus. The three shapes subtended the same area on the display.

Pupil size analysis. We analyzed the event-related pupillary response, time locked to the onset of target stimuli, for each task condition/block. Pupil size (sampled at $60 \mathrm{~Hz}$ together with gaze position) was then analyzed offline using R (R Development Core Team, 2008). Data from the left and right pupil were averaged together, and gaps in the recordings shorter than $100 \mathrm{~ms}$ (due for instance to brief eyeblinks or to eyetracker faults) were linearly interpolated. For each participant and task condition, pupil data were segmented in epochs aligned to target onset. Each epoch was then low-pass filtered with a central moving average filter of 13 samples (at a sampling frequency of $60 \mathrm{~Hz}$ this corresponds to a cut-off frequency of $2 \mathrm{~Hz}$ ), and transformed to differences relative to a baseline level, namely the average pupil size in the $500 \mathrm{~ms}$ before target onset. Only trials with all 
correct responses (both to the primary task and to the additional dual tasks, when required) were included in the pupil size analysis.

\section{Results}

Gaze position recordings were analyzed offline to determine the presence of eye movements. Trials in which the recorded gaze position during the fixation-mask interval deviated from the fixation cross by more than $2^{\circ}$, and for more than $100 \mathrm{~ms}$ consecutively, were excluded from subsequent analysis (4\% of total trials). Trials with gaps in the recordings longer than $100 \mathrm{~ms}$ were excluded ( $2 \%$ of total trials).

Behavioral results. Mean accuracies for the additional tasks (i.e., other than the primary task of reporting lateralized targets position) were $99 \%$ correct for the color task, $94 \%$ for the color-shape task and 92\% for the sound task respectively. Using the lme4 library (Bates \& Sarkar, 2007), available for the open-source software R (R Core Team, 2015), we fitted a generalized mixed-effect model (Pinheiro \& Bates, 2000) with the logistic as link function and the accuracy of responses to dual-tasks as dependent variables; participant was included as random effect factor and task condition as fixed effect predictor. To test the statistical significance of each fixed effect predictor, we compared the change in the residual deviance between the full and a reduced model (i.e., without the selected predictor) using a likelihood ratio test. Results showed a significant effect of task condition $\left[\chi^{2}(2)=68.36, p<0.001\right]$. Specifically, accuracy in the color task was higher compared to both the sound task $[\beta=-2.52, z=-8.24, p<.0001]$ and the shape-color task $[\beta=-$ $2.21, z=-7.16, p<.0001]$. A significant difference also emerged between the sound and shapecolor tasks $[\beta=0.3, z=2.22, p<.05]$.

We then analyzed response accuracy in the primary task (spatial monitoring), excluding from the analysis those trials with wrong responses to the additional tasks (whenever required). We again applied a logistic mixed effects model, with the proportion of correct responses to the lateralized targets as dependent variable. The model included task condition (single, color, shape-color, sound) and lateralized target position (left, right or both) as fixed-effect predictors and participant as random-effect factor. Results showed both a significant effect of task condition $\left[\chi^{2}(3)=17.93, p<\right.$ $0.001]$ and of target position $\left[\chi^{2}(2)=253.69, p<0.001\right]$. The interaction between task condition and target position was not significant $\left[\chi^{2}(6)=11.18, p=0.08\right]$, although there was a trend for a greater decrease in performance with increasing load for the left target condition (see Fig. 2, panel A). The condition with targets appearing in both hemifields (bilateral) yielded a significantly lower accuracy with respect to the left target condition, as indexed by the parameter of the corresponding contrast in the model $[\beta=-1.42, z=-7.89, p<.0001]$. There was no difference between the two unilateral conditions (left vs. right targets) $[\beta=-0.4, z=-0.2, p=0.83]$. It is important to remark that for the current analysis we considered as errors also omissions of a single lateral target in bilateral trials, as well as responses that indicated two targets in unilateral trials. The significant main effect of task condition indexed the decreased accuracy in reporting the lateralized targets when multitasking (see Fig. 2, panel B). Indeed all multitasking conditions yielded a significantly lower accuracy relative to the single task [color, $\beta=-0.66, z=-3.4, p<.001$; color-shape, $\beta=$ $0.75, z=-3.89, p<.001$; sound, $\beta=-0.83, z=-4.31, p<.001]$. The regression coefficients were compared again after switching the contrasts for the 'task condition' factor but no significant differences in accuracy between the three multitasking conditions emerged (all ps $>.05$ ). The latter finding shows that performance level in the spatial task does not reliably discriminate the different types of multitasking conditions. 
A

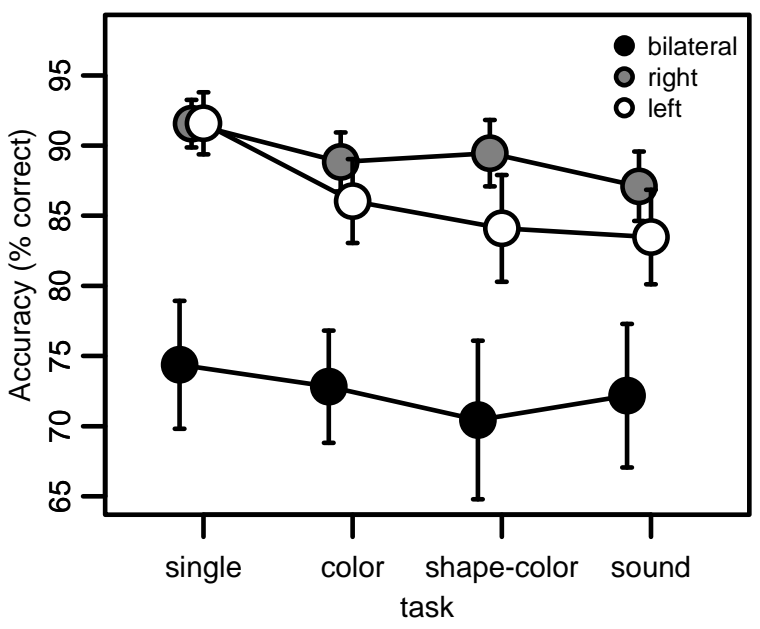

B

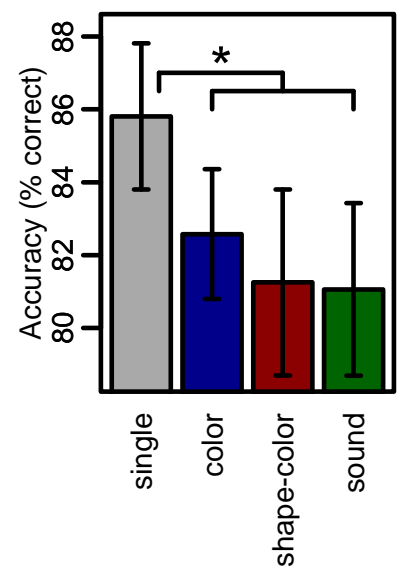

Fig. 2 Behavioral data (A) Mean accuracy in the primary task (spatial monitoring) as a function of target position. (B) Mean accuracy in each task, collapsed across the different target positions. Error bars represent within-subjects SEM (Cousineau, 2005).

Event related pupillary response. Due to the slow time-course of the changes in pupil diameter we took as a measure of pupil dilation the average dilation in the time window between 1.25 and 1.75 seconds after stimulus onset (a window that comprised the maximum dilation in all the four tasks), which was then analyzed as a function of task condition (see Fig. 3, panel A). A repeated-measures ANOVA confirmed a main effect of task on pupil diameter changes $[F(3,69)=$ $14.22, p<.0001]$. Planned comparisons revealed that the color task elicited a significantly greater dilation than the single task $[t(23)=2.46, p=0.02]$. Both the sound and the color-shape tasks elicited a significantly greater dilation than the color task [sound, $t(23)=2.55, p=0.02$; shapecolor, $t(23)=3.30, p=0.003]$, but they did not differ from each other $[t(23)=1.24, p=0.23]$ (all ttest comparisons were paired and two-tailed).

A

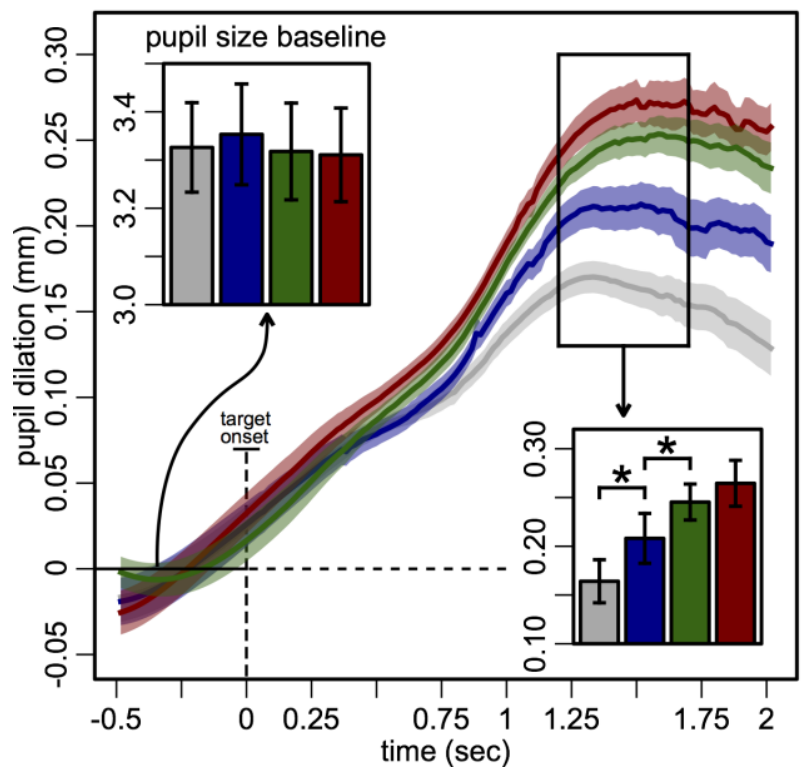

B

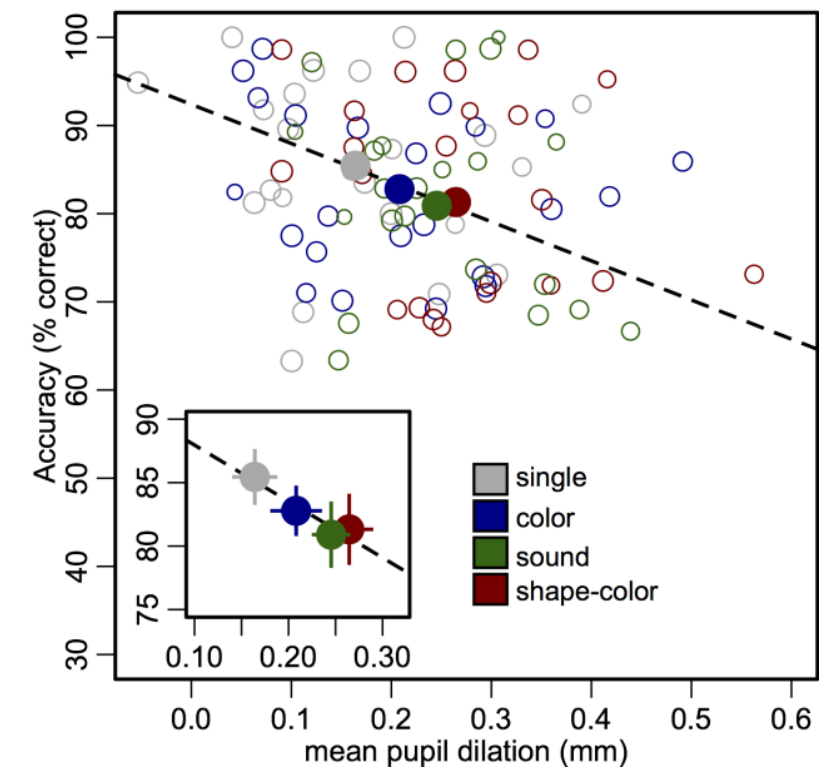

Fig. 3 Pupil dilation data. (A) Time-course of dilation patterns. The top left inset shows mean values of pupil diameter at pre-stimulus baseline (500 ms before target onset), whereas the bottom right inset shows mean pupil dilation in the 1.25-1.75 seconds post-stimulus time window corresponding to the black, top right rectangle superimposed on the dilation time-course. The vertical, dashed line represents target onset. (B) Relation between 
response accuracy to the lateralized targets and pupil dilation across the different tasks (magnified within the inset). Empty dots represent individual data, and filled dots represent group means. Error bands and bars represent SEM.

By comparing pupil data to the accuracy in the primary spatial task, it can be noted that the increase in pupil dilation closely mirrors, at the group level, the reduction in response accuracy (see Fig. 3, panel B). The relation between the two variables is much less evident at the individual level, as a consequence of different task orders and the well-known effect of arousal and fatigue on pupil size (McIntire, McIntire, McKinley, \& Goodyear, 2014; Morad, Lemberg, Yofe, \& Dagan, 2000). Since task order was counterbalanced across participants, the pattern of data can be better appreciated at the group level, where carry-over effects were equated. Indeed the average response accuracy showed a significant negative correlation with peak pupil dilation across the 4 blocks, $r(2)=-.96, p=0.04$, two-tailed. In summary, pupil dilation precisely characterized concurrent processing load during a spatial monitoring task and mirrored the behavioral performance, as indexed by the group-level correlation with the response accuracy in the spatial task. Moreover, pupil dilation also proved to be more sensitive than response accuracy, as it highlighted a significant difference between the color task and both the sound and shape-color tasks; this difference was present as a trend in mean accuracies, but it did not reach statistical significance.

We investigated also whether the amplitude of pupil dilation was larger for bilateral vs. unilateral target. We carried out a repeated-measures ANOVA with the amplitude of pupil dilation as dependent variable, task condition and target number (2, bilateral, vs 1 , unilateral, where unilateral includes both left and right targets) as within-subjects factors. Neither the effect of target number $[F(1,23)=1.49, p=0.23]$, nor the interaction between task and target number $[F(3,69)=$ $0.86, p=0.46]$ were significant. The same analysis performed on the average pupil diameter in the $500 \mathrm{~ms}$ baseline period did not reveal any significant effect (see top left inset in panel A of Fig. 3) [task, $F(3,69)=0.64, p=0.58$; target number, $F(1,23)=1.34, p=0.26$; interaction between task and target number, $F(3,69)=1.08, p=0.36]$.

\section{Hemi-field bias}

Incorrect responses during the spatial monitoring task were further analyzed to explore the role of increased task demands on spatial awareness. This additional analysis was intended to exclude the presence of spatially asymmetric performance, though a strong spatial bias is a-priori unlikely in the absence of brain damage. The probability of a correct response to target position (Fig. 2, panel A) was lower in the bilateral than in the unilateral target condition. To identify a potential hemifield bias we further analyzed the pattern of errors in bilateral trials. A laterality index on the incorrect bilateral trials was computed for each participant and task condition as follows:

$$
\text { laterality index }=\frac{R-L}{R+L}
$$

$R$ and $L$ indicate, respectively, the number of right and left responses given by participants in bilateral trials (i.e., errors). Though the mean index across participants was positive for three out of four conditions, thereby suggesting a rightward bias [single $=0.11$; color $=0.26$; sound $=0.17$; colorshape $=-0.03$ ], all $\mathrm{t}$-tests against zero failed to reach significance (all $p>0.05$ ). A repeated-measures ANOVA with task condition as within subject predictor also did not reveal any significant effect.

\section{Discussion}

We examined whether event-related pupillary responses were sensitive enough to characterize 'pure', top-down, attentional load. We manipulated attentional load across four task conditions by adding one/two concurrent tasks to the primary spatial task (detection of lateralized targets). Importantly, across all single and dual-task conditions the stimulation was kept constant: this was necessary because it is known that each and every stimulus is likely to elicit a pupil dilation response (e.g., Hess \& Polt, 1960; Laeng et al., 2012; Laeng \& Falkenberg, 2007; Partala \& Surakka, 2003). We found that event-related pupil dilation (time locked to stimulus onset) was modulated by the increasing task demands. As expected, the single task yielded the highest accuracy as well as the smallest pupil dilation in comparison to all other task conditions. Spatial monitoring in the color task (which implied dual tasking) was performed with lower accuracy than 
when it was performed in isolation during the single task, and increased pupil dilation paralleled this finding. In turn, the color task produced a lower dilation with respect to the two other tasks (sound and color-shape), which on the contrary did not differ from each other in terms of accuracy. The pattern of results clearly indicates that our top-down manipulation of task difficulty not only resulted in increased error rate in the primary spatial task but also committed participants to greater task engagement, as indexed by the larger stimulus-related pupil dilations. Our experimental design allowed to disentangle processing load from potential confounds like the number of stimuli that had to be responded to and/or the number of alternative responses. Indeed, the 'triple-task' (colorshape) and the sound dual-task conditions elicited a similar pupillary response and also yielded a similar error rate, even though the number of stimuli to be discriminated was greater in the 'triple' task. This finding allows to rule out the mere number of items in working memory (Beatty \& Lucero-Wagoner, 2000; Kahneman \& Beatty, 1966; Kahneman, Peavler, \& Onuska, 1968; Granholm, Asarnow, Sarkin, \& Dykes, 1996) or other response selection/preparation mechanisms as the main determinants of pupil dilation differences. In contrast, it suggests that the two task conditions had similar attentional demands, and therefore yielded similar error rates and pupil dilations. The lack of difference between these two conditions can be explained assuming that color is a feature mostly processed pre-attentively (Treisman \& Souther, 1985). Without challenging those studies showing that attention can be critical even for the detection of the so-called preattentive features (Joseph, Chun, \& Nakayama, 1997), it seems very likely that color discrimination provided much less load on attentional capacity. This interpretation is corroborated by the finding that the color dual task yielded lower pupil dilation than the sound dual task, despite having the same number of stimuli to be discriminated. Even though it is likely that working memory processes contributed to the pupillary dilation, a simple explanation in terms of response rehearsal cannot account for the observed pattern In contrast, the most parsimonious interpretation of the present set of results is that pupil dilation reflected the attentional preparation required to discriminate, across the same or different modalities, simultaneous stimuli. Attention allocation in our paradigm has the scope of optimizing visual and auditory processing in face of uncertainty about location (lateralized targets) and/or identity (sound/shape/color) of task-relevant stimuli.

Overall, the pattern of results can be explained only assuming that our measures (pupil dilation, and error rate in the primary spatial task) truly reflect attentional load. It has been previously shown that more challenging tasks, such as discrimination, elicit larger phasic dilation than easier tasks like simple detection (Gabay et al., 2011). This fits well with the notion that, of the two modes of activity of the LC-NE system, the one engaged during more demanding tasks is characterized by larger phasic activations and larger pupil dilations (Aston-Jones et al., 1999; Corbetta et al., 2008). Our results extend this finding by showing that the amplitude of pupil dilations precisely mirrors increasing levels of top-down attentional load across discrimination tasks of very different nature, and suggest that the phasic mode of the LC-NE system can be activated at different levels in a flexible way according to task demands. The absence of a main effect of target number (unilateral vs bilateral spatial targets in the primary task) does not support the presence of a ceiling effect, because the difference was absent even in the single task condition, which elicited the smallest pupil dilation. This finding indicates that pupil dilation depended mostly on task instructions (constant within a block of trials) rather than on trial by trial characteristics of the stimuli presented. We also found no systematic modulation of pupil size at baseline and across task conditions, which suggests that pupil dilation, but not tonic pupil size, is an accurate measure of task difficulty. However, it is also possible that small differences in tonic pupil size, whenever present, were masked by the concurrent effect of other incidental factors such as pupillary hippus or random fluctuations in the level of alertness.

A second aim of the present study was to explore the consequences of increased attentional load upon spatial monitoring and visuospatial awareness. We focused on trials with bilateral targets, which yielded a higher error rate than trials with unilateral targets, in line with the hypothesis of 
between-hemifield competition in conditions of double simultaneous stimulation (Driver \& Vuilleumier, 2001; Kinsbourne, 1977; Miller, Gochin, \& Gross, 1993). Although an overall positive laterality index somehow suggested that participants missed more frequently the left target, no significant differences emerged across hemispaces. It is important to note that the hemifield attentional bias seems to be strongly dependent on stimuli parameters, like spatial frequency (Proverbio, Zani, \& Avella, 1997) or type of spatial task (Roth \& Hellige, 1998), and task demands, like visual short-term memory (VSTM) load (Sheremata, Bettencourt, \& Somers, 2010). This dependence on task contingencies might be the source of discrepancies in the literature. Studies using a dual-stream rapid serial visual presentation (RSVP) task reported a left visual field advantage (Verleger et al., 2009, 2010; Verleger, Śmigasiewicz, \& Möller, 2011), which could reflect a specific advantage of the right hemisphere for processing fast-paced sequences of stimuli. In contrast, other studies have shown that the attentional bias at baseline turns into a rightward bias following increased cognitive load (Dodds et al., 2008; Peers et al., 2006) and following decreased arousal and alertness (Bellgrove, Dockree, Aimola, \& Robertson, 2004; Fimm, Willmes, \& Spijkers, 2006; Manly, Dobler, Dodds, \& George, 2005; Matthias et al., 2009; Schmitz, Deliens, Mary, Urbain, \& Peigneux, 2011). In our data we did not find any evidence of a modulation of the hemifield bias due to attentional load. One possible explanation is that verbal responses, mediated by the left-hemisphere, could have interfered with the processing of visual stimuli in the righthemifield: according to the 'functional distance model' (Kinsbourne \& Hicks, 1978) the amount of interference in a dual task is dependent upon the distance between the control centers involved in the two concurrent tasks. In other words, tasks that are processed by anatomically close regions are more difficult to perform together (for instance, speaking and using the right hand; Kinsbourne \& Cook, 1971). Our multitasking conditions required an additional verbal response, recruiting more resources in the left hemisphere and perhaps weakening visual processing of the stimuli presented on the right. Thus, it is conceivable that the verbal response could have prevented the rightward bias to increase in the dual task conditions, in contrast to other studies that used manual responses (Peers et al., 2006; Pérez et al., 2009). Further studies are required to clarify the relationship between response modality and the direction of the hemifield attentional bias.

In conclusion, our results show that pupil dilation accurately mirror variations in top-down attentional load in a task where bottom-up confounds were kept constant across conditions, thereby supporting and extending the use of this valuable and easy-measurable psychophysiological index. Pupillometry seems to have the potential to be fruitfully implemented in a variety of settings, both experimental and non-experimental. The possibility to record and categorize performance in the absence of an overt response seems particularly promising for an accurate online monitoring of task load or flexible allocation of attentional resources.

\section{Acknowledgements}

This study was supported by a grant from the University of Padova (Strategic Grant "NEURAT") to MZ. MB was supported by a Marie Curie Intra European Fellowship within the 7th European Community Framework Program. The Authors are grateful to Sara Pegoraro for helping with data collection. 


\section{References}

Ahern, S., \& Beatty, J. (1979). Pupillary responses during information processing vary with Scholastic Aptitude Test scores. Science, 205(4412), 1289-1292.

Aston-Jones, G., \& Cohen, J. D. (2005). An integrative theory of locus coeruleus-norepinephrine function: adaptive gain and optimal performance. Annual Review of Neuroscience, 28, 403450. doi:10.1146/annurev.neuro.28.061604.135709

Aston-Jones, G., Rajkowski, J., \& Cohen, J. (1999). Role of locus coeruleus in attention and behavioral flexibility. Biological Psychiatry, 46(9), 1309-1320.

Aston-Jones, G., Rajkowski, J., Kubiak, P., \& Alexinsky, T. (1994). Locus coeruleus neurons in monkey are selectively activated by attended cues in a vigilance task. Journal of Neuroscience, 14(7), 4467-4480.

Bates, D. M., \& Sarkar, D. (2007). lme4: Linear mixed-effects models using S4 classes.

Beatty, J. (1982). Task-evoked pupillary responses, processing load, and the structure of processing resources. Psychological Bulletin, 91(2), 276-292. doi:10.1037/0033-2909.91.2.276

Beatty, J., \& Lucero-Wagoner, B. (2000). The pupillary system. In J. T. Cacioppo, L. G. Tassinary, \& G. G. Berntson (Eds.), Handbook of psychophysiology (2nd ed., pp. 142-162). New York, NY, US: Cambridge University Press.

Bellgrove, M. A., Dockree, P. M., Aimola, L., \& Robertson, I. H. (2004). Attenuation of spatial attentional asymmetries with poor sustained attention. Neuroreport, 15(6), 1065-1069. doi:10.1097/01.wnr.0000124663.90292.64

Bonato, M. (2012). Neglect and extinction depend greatly on task demands: a review. Frontiers in Human Neuroscience, 6, 195. doi:10.3389/fnhum.2012.00195

Bonato, M. (2015), Unveiling residual, spontaneous recovery from subtle hemispatial neglect three years after stroke. Frontiers in Human Neuroscience, 9:413. doi: 10.3389/fnhum.2015.00413.

Bonato, M., Priftis, K., Marenzi, R., Umiltà, C., \& Zorzi, M. (2010). Increased attentional demands impair contralesional space awareness following stroke. Neuropsychologia, 48(13), 39343940. doi:10.1016/j.neuropsychologia.2010.08.022

Bonato, M., Priftis, K., Umiltà, C., \& Zorzi, M. (2013). Computer-based testing unveils severe neglect in apparently intact patients. Behavioural Neurology, 25, 1-3. doi:10.3233/BEN-2012129005

Bonato M., Spironelli C., Lisi M., Priftis K., \& Zorzi M. (2015). Effects of multimodal load on spatial monitoring as revealed by ERPs. Plos ONE, 10(9), 1-21. doi:

10.1371/journal.pone.0136719

Brown, G. G., Kindermann, S. S., Siegle, G. J., Granholm, E., Wong, E. C., \& Buxton, R. B. (1999). Brain activation and pupil response during covert performance of the Stroop Color Word task. Journal of the International Neuropsychological Society, 5, 308-319. 
Corbetta, M., Miezin, F. M., Shulman, G. L., \& Petersen, S. E. (1993). A PET study of visuospatial attention. Journal of Neuroscience, 13(3), 1202-1226.

Corbetta, M., Patel, G., \& Shulman, G. L. (2008). The reorienting system of the human brain: From environment to theory of mind. Neuron, 58(3), 306-324. doi:10.1016/j.neuron.2008.04.017

Dodds, C. M., van Belle, J., Peers, P. V, Dove, A., Cusack, R., Duncan, J., \& Manly, T. (2008). The effects of time-on-task and concurrent cognitive load on normal visuospatial bias. Neuropsychology, 22(4), 545-552. doi:10.1037/0894-4105.22.4.545

Driver, J., \& Vuilleumier, P. (2001). Perceptual awareness and its loss in unilateral neglect and extinction. Cognition, 79(1-2), 39-88.

Fimm, B., Willmes, K., \& Spijkers, W. (2006). The effect of low arousal on visuo-spatial attention. Neuropsychologia, 44(8), 1261-1268. doi:10.1016/j.neuropsychologia.2006.01.027

Gabay, S., Pertzov, Y., \& Henik, A. (2011). Orienting of attention, pupil size, and the norepinephrine system. Attention, Perception \& Psychophysics, 73(1), 123-129. doi:10.3758/s13414-010-0015-4

Gilzenrat, M. S., Nieuwenhuis, S., Jepma, M., \& Cohen, J. D. (2010). Pupil diameter tracks changes in control state predicted by the adaptive gain theory of locus coeruleus function. Cognitive, Affective \& Behavioral Neuroscience, 10(2), 252-269. doi:10.3758/CABN.10.2.252

Granholm, E., Asarnow, R. F., Sarkin, A. J., \& Dykes, K. L. (1996) Pupillary responses index cognitive resource limitations. Psychophysiology, 33, 457-461.

Hess, E. H., \& Polt, J. M. (1960). Pupil size as related to interest value of visual stimuli. Science, $132(3423), 349-350$.

Hess, E. H., \& Polt, J. M. (1964). Pupil size in relation to mental activity during simple problemsolving. Science (New York, N.Y.), 143(3611), 1190-1192. doi:10.1126/science.143.3611.1190

Jepma, M., \& Nieuwenhuis, S. (2011). Pupil diameter predicts changes in the explorationexploitation trade-off: Evidence for the adaptive gain theory. Journal of Cognitive Neuroscience, 23(7), 1587-1596. doi:10.1162/jocn.2010.21548

Joseph, J. S., Chun, M. M., \& Nakayama, K. (1997). Attentional requirements in a "preattentive" feature search task. Nature, 387(6635), 805-807. doi:10.1038/42940

Kaernbach, C. (1991). Simple adaptive testing with the weighted up-down method. Perception \& Psychophysics, 49(3), 227-229. doi:10.3758/BF03214307

Kahneman, D. (1973). Attention and Effort (Vol. 88, p. 339). Englewood Cliffs, New Jersey: Prentice-Hall inc.

Kahneman, D., \& Beatty, J. (1966). Pupil diameter and load on memory. Science, 154(3756), 15831585.

Kahneman, D., \& Beatty, J. (1967). Pupillary responses in a pitch-discrimination task. Perception \& Psychophysics, 2(3), 101-105. doi:10.3758/BF03210302 
Kahneman, D., Peavler, W. S., \& Onuska, L. (1968). Effects of verbalization and incentive on the pupil response to mental activity. Canadian Journal of Psychology/Revue Canadienne de Psychologie, 22(3), 186-196. doi:10.1037/h0082759

Kinsbourne, M. (1977). Hemi-neglect and hemisphere rivalry. Advances in Neurology, 18, 41-49.

Kinsbourne, M., \& Cook, J. (1971). Generalized and lateralized effects of concurrent verbalization on a unimanual skill. Quarterly Journal of Experimental Psychology, 23(3), 341-345. doi:10.1080/14640746908401828

Kinsbourne, M., \& Hicks, R. E. (1978). Functional cerebral space: A model for overflow, transfer and interference effects in human performance: A tutorial review. In J. Renquin (Ed.), Attention and performance VII (pp. 345-362). Hillsdale, NJ: Lawrence Erlbaum Associates.

Laeng, B., \& Falkenberg, L. (2007). Women's pupillary responses to sexually significant others during the hormonal cycle. Hormones and Behavior, 52(4), 520-530.

doi:10.1016/j.yhbeh.2007.07.013

Laeng, B., Ørbo, M., Holmlund, T., \& Miozzo, M. (2011). Pupillary Stroop effects. Cognitive Processing, 12, 13-21.

Laeng, B., Sirois, S., \& Gredeback, G. (2012). Pupillometry: A window to the preconscious? Perspectives on Psychological Science, 7(1), 18-27. doi:10.1177/1745691611427305

MacLachlan, C., \& Howland, H. C. (2002). Normal values and standard deviations for pupil diameter and interpupillary distance in subjects aged 1 month to 19 years. Ophthalmic \& Physiological Optics, 22(3), 175-182.

Manly, T., Dobler, V. B., Dodds, C. M., \& George, M. A. (2005). Rightward shift in spatial awareness with declining alertness. Neuropsychologia, 43(12), 1721-1728. doi:10.1016/j.neuropsychologia.2005.02.009

Matthias, E., Bublak, P., Costa, A., Müller, H. J., Schneider, W. X., \& Finke, K. (2009). Attentional and sensory effects of lowered levels of intrinsic alertness. Neuropsychologia , 47(14), 32553264. doi:10.1016/j.neuropsychologia.2009.08.004

McIntire, L. K., McIntire, J. P., McKinley, R. A., \& Goodyear, C. (2014). Detection of vigilance performance with pupillometry. In Proceedings of the Symposium on Eye Tracking Research and Applications - ETRA '14 (pp. 167-174). New York, New York, USA: ACM Press. doi: $10.1145 / 2578153.2578177$

Mesulam, M. M. (1981). A cortical network for directed attention and unilateral neglect. Annals of Neurology, 10(4), 309-325. doi:10.1002/ana.410100402

Miller, E. K., Gochin, P. M., \& Gross, C. G. (1993). Suppression of visual responses of neurons in inferior temporal cortex of the awake macaque by addition of a second stimulus. Brain Research, 616(1-2), 25-29.

Morad, Y., Lemberg, H., Yofe, N., \& Dagan, Y. (2000). Pupillography as an objective indicator of fatigue. Current Eye Research, 21(1), 535-542. 
Murphy, P. R., Robertson, I. H., Balsters, J. H., \& O’Connell, R. G. (2011). Pupillometry and P3 index the locus coeruleus-noradrenergic arousal function in humans. Psychophysiology, 48(11), 1532-1543. doi:10.1111/j.1469-8986.2011.01226.x

Nunnally, J. C., Knott, P. D., Duchnowski, A., \& Parker, R. (1967). Pupillary response as a general measure of activation. Perception \& Psychophysics, 2(4), 149-155. doi:10.3758/BF03210310

O’Connell, R. G., Schneider, D., Hester, R., Mattingley, J. B., \& Bellgrove, M. A. (2011). Attentional load asymmetrically affects early electrophysiological indices of visual orienting. Cerebral Cortex, 21(5), 1056-1065. doi:10.1093/cercor/bhq178

Partala, T., \& Surakka, V. (2003). Pupil size variation as an indication of affective processing. International Journal of Human-Computer Studies, 59(1-2), 185-198. doi:10.1016/S10715819(03)00017-X

Peers, P. V, Cusack, R., \& Duncan, J. (2006). Modulation of spatial bias in the dual task paradigm: evidence from patients with unilateral parietal lesions and controls. Neuropsychologia, 44(8), 1325-1335. doi:10.1016/j.neuropsychologia.2006.01.033

Pérez, A., Peers, P. V, Valdés-Sosa, M., Galán, L., García, L., \& Martínez-Montes, E. (2009). Hemispheric modulations of alpha-band power reflect the rightward shift in attention induced by enhanced attentional load. Neuropsychologia, 47(1), 41-49. doi:10.1016/j.neuropsychologia.2008.08.017

Petersen, S. E., \& Posner, M. I. (2012). The attention system of the human brain: 20 years after. Annual Review of Neuroscience, 35, 73-89. doi:10.1146/annurev-neuro-062111-150525

Pinheiro, J. C., \& Bates, D. M. (2000). Mixed-Effects Models in S and S-PLUS. New York: Springer-Verlag. doi:10.1007/b98882

Posner, M. I., \& Petersen, S. E. (1990). The attention system of the human brain. Annual Review of Neuroscience, 13, 25-42. doi:10.1146/annurev.ne.13.030190.000325

Proverbio, A. M., Zani, A., \& Avella, C. (1997). Hemispheric asymmetries for spatial frequency discrimination in a selective attention task. Brain and Cognition, 34(2), 311-320. doi:10.1006/brcg.1997.0901

R Core Team. (2015). $R$ : A language and environment for statistical computing. Vienna, Austria: $\mathrm{R}$ Foundation for Statistical Computing. URL: https://www.R-project.org/

Roth, E. C., \& Hellige, J. B. (1998). Spatial processing and hemispheric asymmetry. Contributions of the transient/magnocellular visual system. Journal of Cognitive Neuroscience, 10(4), 472484.

Samuels, E. R., \& Szabadi, E. (2008). Functional neuroanatomy of the noradrenergic locus coeruleus: Its roles in the regulation of arousal and autonomic function part I: Principles of functional organisation. Current Neuropharmacology, 6(3), 235-253. doi:10.2174/157015908785777229 
Schmitz, R., Deliens, G., Mary, A., Urbain, C., \& Peigneux, P. (2011). Selective modulations of attentional asymmetries after sleep deprivation. Neuropsychologia, 49(12), 3351-3360. doi:10.1016/j.neuropsychologia.2011.08.009

Sheremata, S. L., Bettencourt, K. C., \& Somers, D. C. (2010). Hemispheric asymmetry in visuotopic posterior parietal cortex emerges with visual short-term memory load. The Journal of Neuroscience, 30(38), 12581-12588. doi:10.1523/JNEUROSCI.2689-10.2010

Treisman, A., \& Souther, J. (1985). Search asymmetry. A diagnostic for preattentive processing of separable features. Journal of Experimental Psychology: General, 114(3), 285-310.

Usher, M. (1999). The role of locus coeruleus in the regulation of cognitive performance. Science, 283(5401), 549-554. doi:10.1126/science.283.5401.549

van Steenbergen, H., \& Band, G. P. (2013). Pupil dilation in the Simon task as a marker of conflict processing. Frontiers in Human Neuroscience, 7:215.

Verleger, R., Möller, F., Kuniecki, M., Smigasiewicz, K., Groppa, S., \& Siebner, H. R. (2010). The left visual-field advantage in rapid visual presentation is amplified rather than reduced by posterior-parietal rTMS. Experimental Brain Research, 203(2), 355-365. doi:10.1007/s00221$010-2237-z$

Verleger, R., Śmigasiewicz, K., \& Möller, F. (2011). Mechanisms underlying the left visual-field advantage in the dual stream RSVP task: Evidence from N2pc, P3, and distractor-evoked VEPs. Psychophysiology, 48(8), 1096-1106. doi:10.1111/j.1469-8986.2011.01176.x

Verleger, R., Sprenger, A., Gebauer, S., Fritzmannova, M., Friedrich, M., Kraft, S., \& Jaśkowski, P. (2009). On why left events are the right ones: Neural mechanisms underlying the lefthemifield advantage in rapid serial visual presentation. Journal of Cognitive Neuroscience, 21(3), 474-488. doi:10.1162/jocn.2009.21038

Wilhelm, B., Wilhelm, H., \& Lüdtke, H. (1999). Pupillography: Principles and applications in basic and clinical research. In J. Kuhlmann \& M. Böttcher (Eds.), Pupillography: Principles, methods and applications (pp. 1-11). München, Germany: Zuckschwerdt Verlag. 